\title{
The Holy Grail in diagnostic neuroradiology: 3T or 3D?
}

\author{
Frederik Barkhof • Petra J. W. Pouwels • \\ Mike P. Wattjes
}

Received: 29 November 2010 /Revised: 2 December 2010 / Accepted: 7 December 2010/Published online: 23 December 2010

(C) The Author(s) 2010. This article is published with open access at Springerlink.com

\begin{abstract}
Many technical developments keep occurring in the field of MRI that could benefit image acquisition in the field of diagnostic neuroradiology. While there is much focus on the potential advantages of $3 \mathrm{~T}$ and higher field strengths, it is often unclear whether these are cosmetic only, or convey clinically relevant diagnostic value. The increased signal-to-noise at $3 \mathrm{~T}$ is certainly beneficial in different ways particularly for the acquisition of isotropic 3D sequences like FLAIR. Single-slab 3D sequences can now be obtained with multiple contrasts in clinically attainable data acquisition times and could revolutionize MRI to evolve into a fundamentally multi-planar technique, rather similar to what has happened with the introduction of multi-detector row CT.
\end{abstract}

\section{The many revolutions of MRI}

The invention and development of MRI as a diagnostic tool itself is considered by many one of the greatest medical achievements of the past decades [1]. In retrospect is hard to understand the enthusiasm of the early days given the quality of the initial MR images published. Those were $9 \mathrm{~mm}$ slices of the brain with an in-plane resolution of $2 \times 2 \mathrm{~mm}^{2}$, with $\sim 10$ slices acquired at 0.15 Tesla over $10 \mathrm{~min}$ or more [2].

F. Barkhof $(\varangle) \cdot$ P. J. W. Pouwels $\cdot$ M. P. Wattjes

Department of Radiology,

VU University Medical Center,

P.O. Box 7057, 1008 MB, Amsterdam, The Netherlands

e-mail: f.barkhof@vumc.nl

P. J. W. Pouwels

Department of Physics \& Medical Technology,

VU University Medical Center,

P.O. Box 7057, 1008 MB, Amsterdam, The Netherlands
Many improvements have since occurred at a rapid pace, including stronger magnets and gradients, improved receiver coils, and faster and more sensitive pulse-sequences; overall leading to faster and better image acquisition. Most popular developments were aiming for a higher signal-tonoise ratio (SNR). The field strength increased from $0.15 \mathrm{~T}$ to $3 \mathrm{~T}$ or higher, while gradient strength increased up to $40 \mathrm{mT} / \mathrm{m}$ or more enabling imaging at higher spatial resolution. Signal reception was improved by development of multi-array coils, allowing parallel imaging, which in combination with RARE (rapid acquisition with relaxation enhancement) led to much shorter acquisition times.

Evidently, these technical improvements mystify the practice of neuroradiology and request a constant reflection of the optimal combination of imaging parameters. Having a 3T MR system is attractive to patients and doctors alike, but often without good reason-just like a sports-car is more attractive than a regular family car. Even though the costs of 3T MR systems might be approximating those of $1.5 \mathrm{~T}$ machines, it is important to consider what it is that we want to achieve. Is it just prettier images, better spatial resolution, faster acquisition times, or perhaps something else? In the following we will argue that obtaining isotropic 3D data-sets is one of the major quests in neuroradiology.

\section{Advantages of 3T: diagnostic relevant or cosmetic imaging?}

Since the FDA approval in 2000, the installed base of wholebody high-field MR systems operating at 3T for clinical purposes has increased dramatically. Driven by the increased SNR which is the most obvious advantage of higher magnetic field strengths, many studies have demonstrated the potential benefit of $3 \mathrm{~T}$ compared to $1.5 \mathrm{~T}$ mainly in field of Neuroradi- 
ology [3, 4]. The higher SNR can be invested in two different ways: to increase spatial resolution or to decrease acquisition times, or a combination of both. However, the straightforward advantage in terms of higher SNR is substantially counterbalanced by several other factors such as the increased specific absorption rate (SAR), magnetic susceptibility effects, radiofrequency field inhomogeneity and magnetic shielding effects. These factors make imaging at higher field strengths more challenging, although most problems (SAR, susceptibility) can be overcome by techniques such as parallel imaging.

The advantages of $3 \mathrm{~T}$ over $1.5 \mathrm{~T}$ in the field of Neuroradiology have been demonstrated for several clinical applications such as structural brain imaging, e.g. multiple sclerosis (MS), brain tumours, epilepsy, magnetic resonance angiography (MRA) but also quantitative MR applications such as diffusion-weighted/diffusion-tensor imaging, MR perfusion (contrast-enhanced and arterial-spin labelling), MR spectroscopy and functional MRI [5-13]. However, the crucial question remains whether these advantages do have any clinical relevance. In other words, does $3 \mathrm{~T}$ lead to an earlier and more specific diagnosis in neuroradiology? To address this question, the diagnostic value of $3 \mathrm{~T}$ MRI has been extensively studied in MS patients. Although 3T MRI detected significantly more inflammatory lesions in MS patients particularly in anatomical areas which are very important for the diagnosis of MS, 3T did not lead to an earlier diagnosis of MS [5-8]. Another example is MRA. Beyond doubt, the image quality of contrast-enhanced and time-of-flight (TOF) MRA at 3T benefits from the higher SNR and enables the visualization of more peripheral arterial branches [9-11]. However, the question remains whether small aneurysms or a residual post-coiling aneurysmal neck detected by $3 \mathrm{~T}$ MR and not at $1.5 \mathrm{~T}$ have any therapeutic consequences [14]. The increase in SNR is most advantageous in low contrast sequences such as diffusiontensor imaging $[15,16]$.

Finally we have to be aware that magnetic field strength is only one way to turn the screw upwards for a better and faster image acquisition. The application of multi-channel coil technologies both for RF reception and RF transmission at standard field strengths can improve the image quality without dealing with any drawbacks related to higher magnetic fields.

\section{The quest for isotropic resolution}

In a current typical MRI examination, multiple stacks of 2D images, each providing a different tissue contrast, are acquired in multiple planes. Each of the sequences provides independent information (e.g. T1, T2, or susceptibility weighting) and each imaging plane allows the pathology to be depicted in a different relationship with relevant anatomical features. Take the example of a brain tumour, where one would like to capture its extension in relation to important anatomical landmarks, some of which are seen well in the sagittal plane (e.g. the corpus callosum, others better in the coronal plane (e.g. the hippocampus).

One of the bizarre features of MRI remains the anisotropic nature of data-collection - inherent to most 2D imaging protocols. A routine brain examination will often use 3$5 \mathrm{~mm}$ slice thickness with an in-plane resolution of 0.5 $1.0 \mathrm{~mm}$, resulting in highly anisotropic resolution. This probably reflects the battle between anatomical resolution and maintenance of SNR (per time-unit) that can be achieved with 2D sequences - obtaining more slices per TR is unattainable.

In fact, the anisotropic voxel dimensions are undesirable for many reasons, most importantly because they preclude reformatting of data in a plane different from the original one without severe penalties. Such limitations can be overcome by the use of $3 \mathrm{D}$ sequences with isotropic spatial resolution. The need for a second phase-encoding direction, however poses a severe limitation. Only recently have the combination of parallel imaging and RARE with very long echo-trains allowed the development and successful implementation of single-slab 3D sequences.

\section{Maximizing contrast opportunities in 3D}

Initially only fast sequences like gradient-echo could be acquired in 3D mode, due to the short echo-time inherent to the absences of a refocusing $180^{\circ}$ pulse. Very successful examples include 3D heavily T1-weighted sequences like MP-RAGE/FSPGR and MR angiography. More challenging have been T2-weighted sequences. For myelographic purpose very heavily T2-weighed sequences like CISS/FIESTA have been developed, again based on (steady-state) gradient echo.

Initial experience with 3D-FLAIR was based on multi-slab implementation: even though acquisition times were prohibitively long, their advantage in terms of better lesion detection and more homogeneous CSF suppression was obvious [17, 18]. More recently, single-slab 3D spin-echo based image acquisition has been established by the usage of very long echo-trains in combination with variable refocusing flip angles [19]. The flip angles are prospectively designed to establish a pseudo-steady-state at the beginning of the echo train, maintaining constant signal intensity over a certain portion of the echo-train, and decreasing exponentially towards the end. Using this approach the effective TE (and extent of T2-weighting) is much smaller than expected from such long echo-trains. Moreover, the long TR that is needed for T2-weighted imaging is efficiently used, allowing 3D isotropic datasets to be acquired within acceptable scans times, certainly in combination with parallel imagingfacilitated by multi-array coils and attainable best at higher field (especially $3 \mathrm{~T}$ and higher). 
Several sequences, each providing different contrast between important structures, can be obtained with these 3D single-slab techniques (Fig. 1). Acquisition of a heavily T2weighted 3D dataset is feasible within a few minutes. Due to the presence of an inversion time to attenuate the CSF signal the acquisition time of a 3D-FLAIR is slightly longer. However, using parallel imaging, a 3D-FLAIR dataset with $1.0-1.2 \mathrm{~mm}$ isotropic resolution can be obtained within
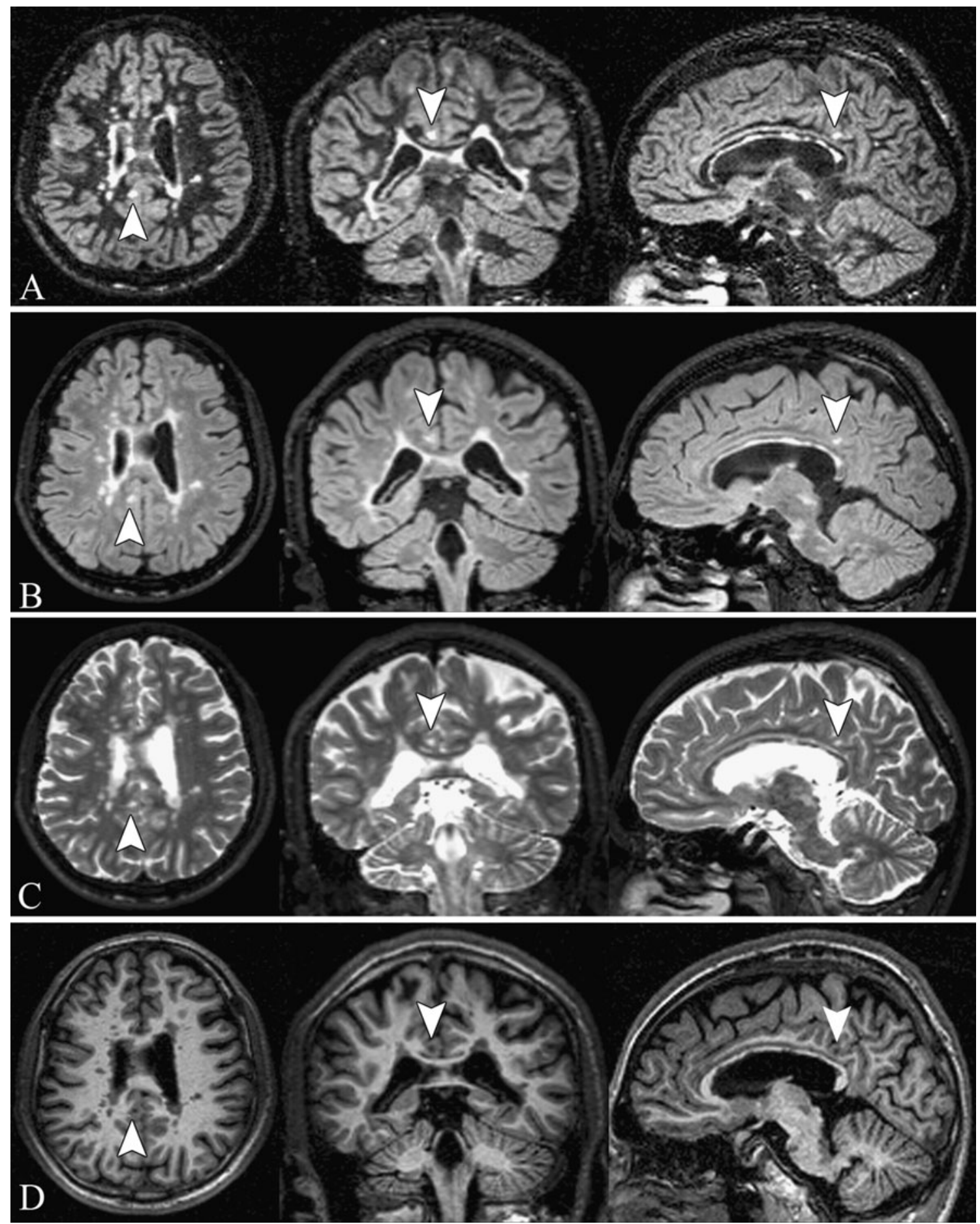

Fig. 1 Multiplanar reformats from multi-contrast isotropic MRI in an MS patient. a: 3D-DIR, b: 3D-FLAIR, c: 3D-T2 and d: 3DMPRAGE. First and second column show axial and coronal reformatted MR images respectively, third column shows original sagittal images. Due to the near isotropic resolution, image quality is preserved in all orientations. Note the (intra)cortical lesion in the right posterior cingulate gyrus (arrowheads). Image kindly provided by Bastiaan Moraal 
5 min, even at $1.5 \mathrm{~T}$. The 3D-FLAIR sequence is extremely useful in a clinical setting, for instance to delineate tumours, MS lesions, and vascular white matter changes. An additional inversion pulse transforms the 3D-FLAIR into a 3D-DIR (double inversion recovery) sequence. "Grey-matter only" images are obtained when the combination of two inversion times are optimized to attenuate both CSF and white matter on the basis of their T1-difference with gray matter [20]. Lesions turn out as hyperintense due to T2weighting during the read-out, and the method is especially sensitive to cortical lesions [21]. Because the second inversion time also partly destroys magnetization of gray matter, the images suffer from a relatively low SNR. Therefore, especially the 3D-DIR technique benefits from higher field strength.
Fig. 3 Use of non-linear image registration in Alzheimer's disease. Baseline MR examination (left), repeat MR examination (middle) and colour overlay overlaid on the baseline examination (right) of four individual patients: a 50-year-old control subject, who presented at the memory clinic with subjective memory complaints (a); a 72-year-old MCI patient who remained stable during follow-up (b); a 69-year-old MCI patient who progressed to $\mathrm{AD}$ during follow-up (c); a 64-yearold, moderately demented AD patient (d). Green and blue represent moderate to severe contraction (atrophy), yellow and red moderate to severe expansion. Image kindly provided by Jasper Sluimer

\section{Clinical applications of 3D imaging}

The isotropic properties of single-slab 3D images can be exploited in all kinds of reconstruction algorithms. Standard multi-planar reconstruction of 3D-T1 and 3DFLAIR images can be used to visualize pathologies
Fig. 2 Glioma progression. This patient with an initially low grade glioma was followed with 3D-FLAIR over 6 monthly intervals after surgery. At baseline, small areas of tumour are visible in the left frontal lobe, which had clearly progressed at follow-up. The upper row shows original sagittal acquired 3D-FLAIR images, the lower row shows transverse reformats available without additional further data-acquisition

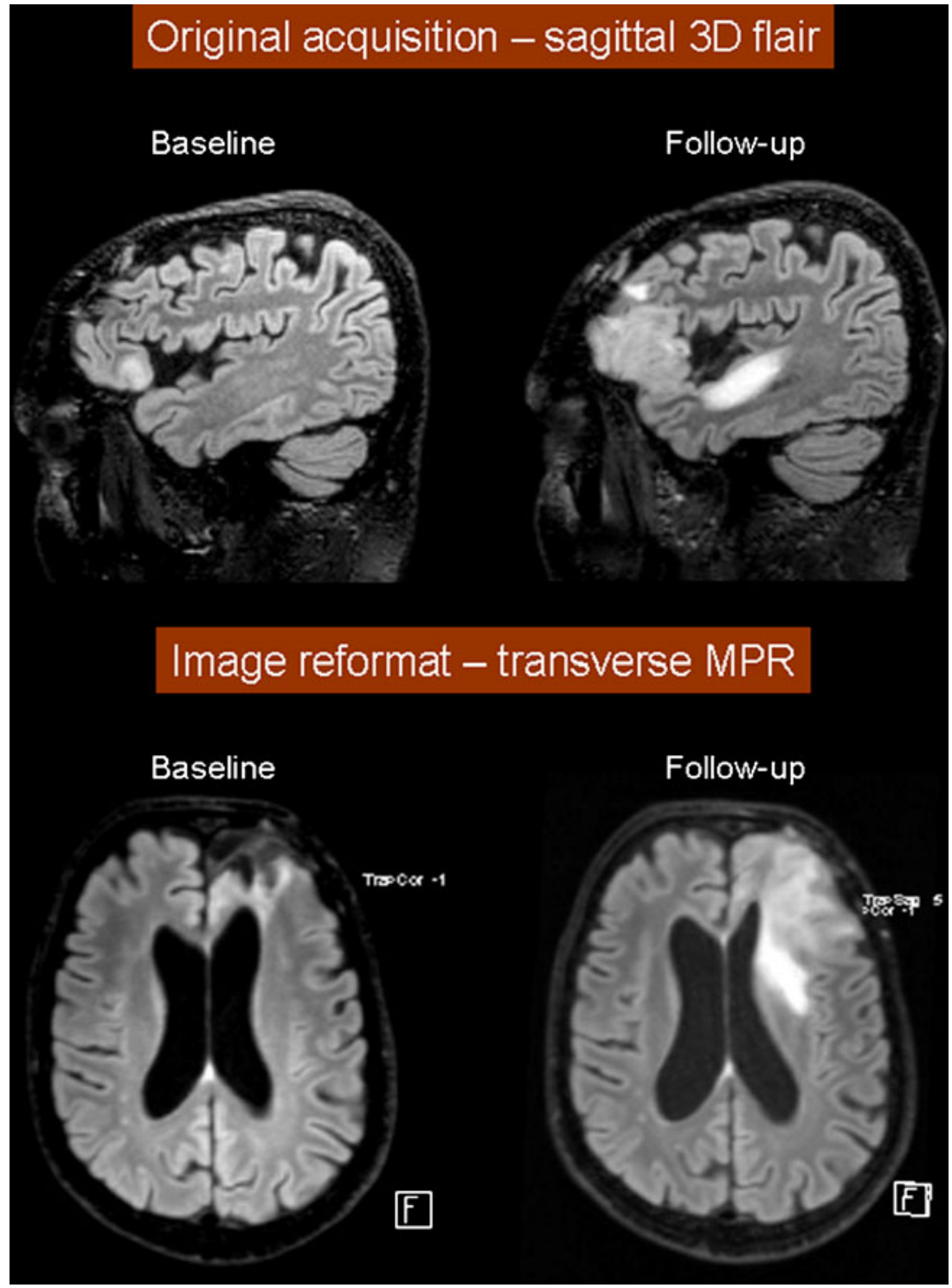



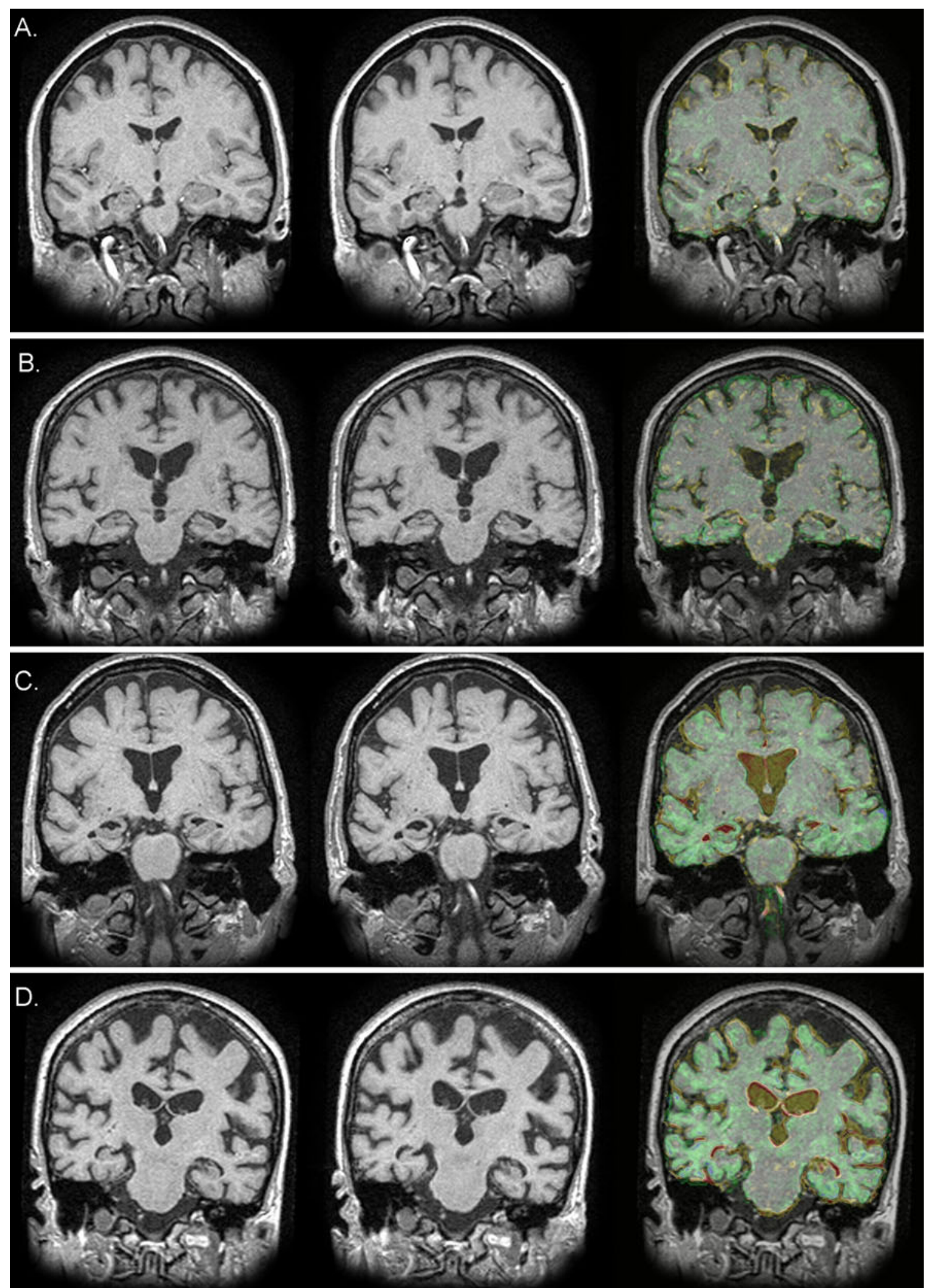

contracting

expanding 

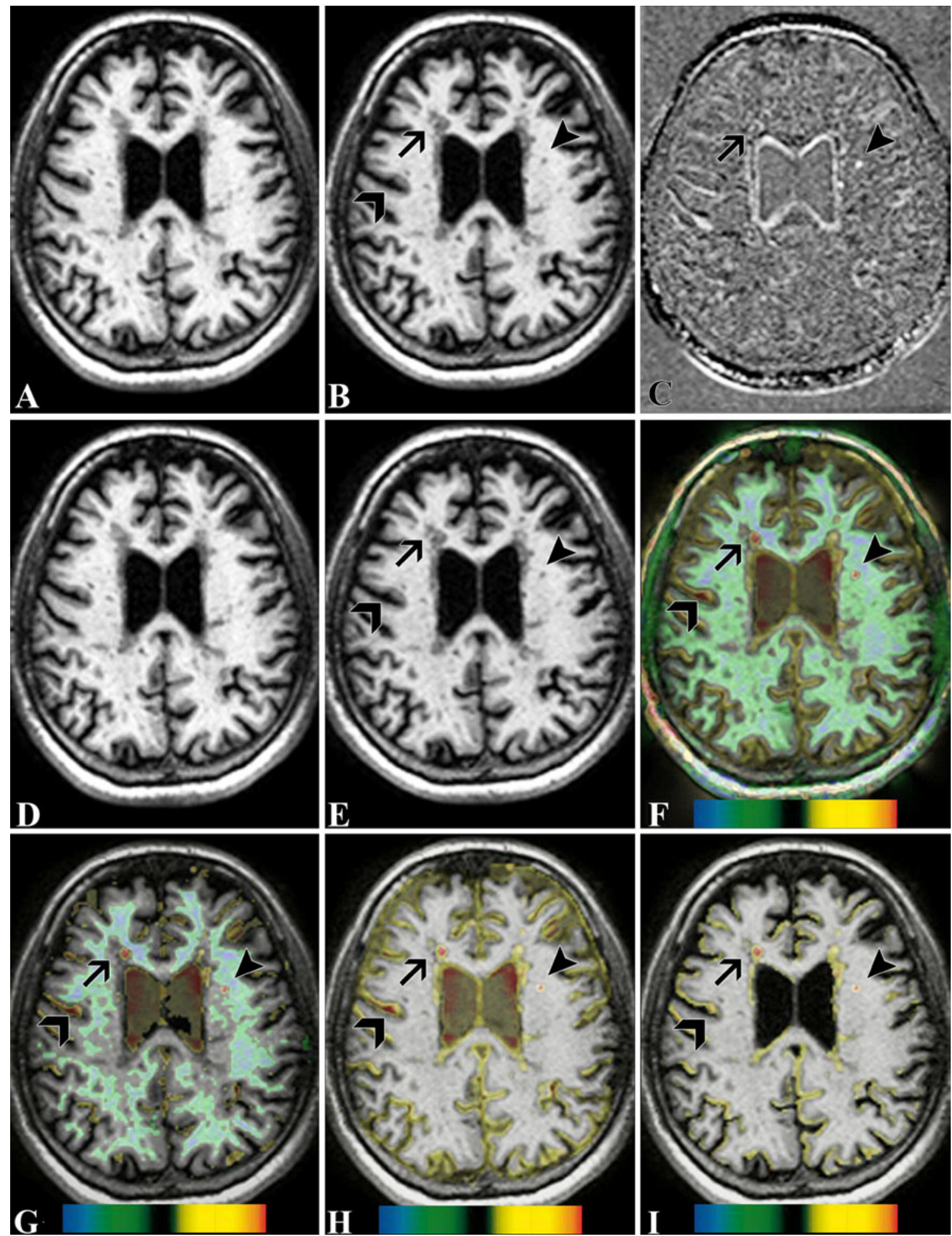

Fig. 4 Non-linear registration to detect MS disease activity over 3 years. a,d: halfway-registered baseline 3D-MPRAGE image; b: rigid-body halfway registered follow-up 3D-MPRAGE image; c: rigid-body subtraction image; e: FLUID registered follow-up image; f,g,h,i: halfway-registered baseline 3D-MPRAGE images overlaid with various FLUID stretch-maps. Colour bars indicate up to $20 \%$ contraction (green to blue) or up to $20 \%$ expansion (yellow to orange) per voxel. f: normal stretch-map; g: showing only activity greater than $10 \%$; $\mathbf{h}$ : showing only expansion; i: showing only expansion within brain parenchyma mask. Arrowheads: New lesion; Arrows: Possible disease activity within a pre-existing lesion; Delta arrows: CSF expansion hampering the detecting of active lesions, which are almost nullified by displaying only expanding voxels within the brain parenchyma mask. Image kindly provided by Bastiaan Moraal 
routinely in multiple orientations. For instance, a coronal reconstruction of a 3D-FLAIR will be used in medial temporal sclerosis, whereas the growth of a tumour will best be appreciated when visualized in 3 orientations (Fig. 2). Using 3D-FLAIR, the cortex may be visualized for pre-surgical purposes with $3 \mathrm{D}$ rendering techniques [22]. An even more realistic pre-surgical picture is obtained with an overlay of superficial arteries and veins, obtained with 3D imaging following intravenous administration of contrast agent.

Currently, 3D T1-weighted images (with or without contrast enhancement) are typically used in presurgical planning or planning of stereotactic radiotherapy, but an additional sequences, such as 3D-FLAIR, may add valuable information particularly in terms of non-enhancing tumour components. A main advantage of these $3 \mathrm{D}$ images is that they are easily registered and displayed within the same geometrical frame.

\section{Towards 4D imaging and beyond}

The isotropic character of these 3D images also allows a registration of longitudinally obtained data, with only minimal blurring effects due to interpolation. Within subject registration of 3D T1-weighted images already has shown great promise in patients suspected of dementia (Fig. 3) and could provide additional information in white matter disease as well (Fig. 4). Registration to a brain template and segmentation into cortex, white matter and CSF is currently performed mainly on 3D T1-weighted images. However, the presence of lesions introduces problems in automatic segmentation, because lesions represent an additional tissue type, which is not easily distinguished from CSF or cortex when using only one 3D sequence with just one type of contrast. A multi-contrast 3D dataset (Fig. 1) should greatly facilitate automatic detection of pathological tissue classes, including tumours and white matter lesions.

\section{Limitations to be overcome}

With currently available 3D techniques, high-resolution $1 \mathrm{~mm}^{3}$ isotropic data-sets can be obtained in approximately 3 to $10 \mathrm{~min}$ (in the order 3D-T2, 3D-FLAIR, 3D-T1 to 3DDIR; depending on field strength and coil configuration). To maximize patient comfort and to achieve a high throughput through an MR Unit, we generally aim to minimize the total necessary time of any clinical MR examination. The potential combination of different levels of contrast within one imaging sequence (similar to Pd-T2 in spin-echo experiments) would be desirable, but does not seem realistic for these $3 \mathrm{D}$ sequences due to the single-slab character and the long echo trains. Using the currently available single-contrast techniques, one should carefully select a unique combination of sequences and MR parameters to maximise the contrast between normal and abnormal tissue, to allow unambiguous differentiation of a lesion.. Thus, one has to decide which combination of 3D datasets is appropriate for a certain indication with the minimum of redundancy (principle of parsimony).

While the 3D images are certainly suitable for registration purposes, one needs to be aware of possible geometric distortions - due to the use of non-linear gradients. Qualitative assessments are not too much influenced by them, but small differences in position of a subject within the same scanner, let alone a subject examined on different MR systems, could have major effects on geometry if not corrected for.

In the current practice, registration of longitudinal datasets, possibly followed by subtraction, is done off-line on separate work-stations and using a variety of software packages. Consequently, this is restricted to a research setting, and the resulting data (subtraction images for instance) are typically unavailable on the central PACS system. Ideally, these post-processing steps should be available on the MR console or satellite workstation. Only then, will the clinical usefulness of using sequential 3D data-sets become a practical reality and thereby allow the full integration of 4D imaging into the clinical arena.

Open Access This article is distributed under the terms of the Creative Commons Attribution Noncommercial License which permits any noncommercial use, distribution, and reproduction in any medium, provided the original author(s) and source are credited.

\section{References}

1. Lauterbur PC (1973) Image formation by induced local interactions: examples of employing nuclear magnetic resonance. Nature 242:190-191

2. Young IR, Hall AS, Pallis CA, Legg NJ, Bydder GM, Steiner RE (1981) Nuclear magnetic resonance imaging of the brain in multiple sclerosis. Lancet 2:1063-1066

3. Schick F (2005) Whole-body MRI at high field: technical limits and clinical potential. Eur Radiol 15:946-959

4. Willinek WA, Schild HH (2008) Clinical advantages of 3.0T MRI over 1.5T. Eur J Radiol 65:2-14

5. Wattjes MP, Lutterbey GG, Harzheim M et al (2006) Higher sensitivity in the detection of inflammatory brain lesions in patients with clinically isolated syndromes suggestive of multiple sclerosis using high field MRI: an intraindividual comparison of 1.5T with 3.0T. Eur Radiol 16:2067-2073

6. Bachmann R, Reilmann R, Schwindt W, Kugel H, Heindel W, Krämer S (2006) FLAIR imaging for multiple sclerosis: a comparative MR study at 1.5 and 3.0 Tesla. Eur Radiol 16:915-921

7. Simon B, Schmidt S, Lukas C et al (2010) Improved in vivo detection of cortical lesions in multiple sclerosis using double inversion recovery MR imaging at 3 Tesla. Eur Radiol 20:1675-1683 
8. Wattjes MP, Barkhof F (2009) High field MRI in the diagnosis of multiple sclerosis: high field -high yield? Neuroradiology $51: 279-292$

9. Vargas MI, Nguyen D, Viallon M et al (2010) Dynamic MR angiography (MRA) of spinal vascular diseases at 3T. Eur Radiol 20:2491-2495

10. Willinek WA, Born M, Simon B et al (2003) Time-of-flight MA angiography: comparison of 3.0T-imaging and 1.5T-imaginginitial experience. Radiology 229:913-920

11. Willinek WA, Bayer T, Gieseke $J$ et al (2007) High spatial resolution contrast-enhanced MR angiography oft he supraaortal arteries using the quadrature head coil at 3.0T: a feasibility study. Eur Radiol 17:618-625

12. Kakeda S, Korogi Y, Hiai Y et al (2007) Detection of brain metastasis at 3T: comparison among SE, IR-FSE and 3D-GRE sequences. Eur Radiol 17:2345-2351

13. Bink A, Schmitt M, Gaa J, Mugler JP 3rd, Lanfermann H, Zanella FE (2006) Detection of lesions in multiple sclerosis by 2D FLAIR and single-slab 3D FLAIR sequences at 3.0T: initial results. Eur Radiol 16:1104-1110

14. Schaafsma JD, Velthuis BK, Majoie CB et al (2010) Intracranial aneurysms treated with coil placement: test characteristics of follow-up MR angiography-multicenter study. Radiology 256:209-218

15. Kamali A, Kramer LA, Butler IJ, Hasan KM (2009) Diffusion tensor tractography of the somatosensory system in the human brainstem: initial findings using high isotropic spatial resolution at 3.0T. Eur Radiol 19:1480-1488

16. Poser BA, Koopmans PJ, Witzel T, Wald LL, Barth M (2010) Three dimensional echo-planar imaging at 7 Tesla. Neuroimage 51:261-266

17. Tan IL, Pouwels PJ, van Schijndel RA, Adèr HJ, Manoliu RA, Barkhof F (2002) Isotropic 3D fast FLAIR imaging of the brain in multiple sclerosis patients: initial experience. Eur Radiol 12:559-567

18. Naganawa S, Koshikawa T, Nakamura T et al (2004) Comparison of flow artifacts between 2D-FLAIR and 3D-FLAIR sequences at 3T. Eur Radiol 14:1901-1908

19. Mugler JP 3rd, Bao S, Mulkern RV et al (2000) Optimized singleslab three-dimensional spin-echo MR imaging of the brain. Radiology 216:891-899

20. Pouwels PJ, Kuijer JP, Mugler JP 3rd, Guttmann CR, Barkhof F (2006) Human gray matter: feasibility of single-slab 3D double inversion-recovery high-spatial-resolution MR imaging. Radiology $241: 873-879$

21. Moraal B, Roosendaal SD, Pouwels PJ et al (2008) Multi-contrast, isotropic, single-slab 3D MR imaging in multiple sclerosis. Eur Radiol 18:2311-2320

22. Willemse RB, Pouwels PJ, Barkhof F, Vandertop WP (2010) Localisation of the central sulcus region in glioma patients with three-dimensional fluid-attenuated inversion recovery and volume rendering: comparison with functional and conventional magnetic resonance. Br J Neurosurg. doi:10.3109/02688697.2010.522742 